\section{Low Copy Repeat}

\section{J. Arnemann}

Abteilung Molekulargenetik, Labor Dr. Wisplinghoff, Köln, Deutschland

Synonym(e) mäßig wiederholte DNA-Sequenzen

Englischer Begriff low copy repeats (LCRs)

Definition Als Low Copy Repeats (LCR) bezeichnet man in der humanen genomischen DNA Sequenzabschnitte von 1-400 kb Länge, die dupliziert und mit sehr hoher Homologie (>90\%) an einer oder mehreren Stellen im Genom vorkommen.

Beschreibung Evolutionär haben sich die Low Copy Repeats durch segmentale Duplikationen von DNA-Abschnitten während der Genomevolution der Primaten entwickelt. Diese Low Copy Repeats lokalisieren in Genomabschnitten des Menschen, die durch eine chromosomale Instabilität und durch Bruchpunkte von evolutionären Chromosomenrearrangements charakterisiert sind. Ungefähr $5 \%$ der humanen DNA bestehen aus Low Copy Repeats.

Low Copy Repeats werden kausal mit der Entstehung von über 25 genomischen Erkrankungen in Verbindung gebracht.
So wurde in zahlreichen Studien publiziert, dass Low Copy Repeats, die einen intervenierenden Lokus flankieren, eine illegale nicht homologe Rekombination eingehen können und es als Ergebnis dieses Prozesses zur Deletion, Duplikation oder Inversion dieser intervenierenden Sequenz kommen kann. Diese Rearrangements müssen nicht zwingend einen pathogenen Effekt haben, sondern können auch einen genomischen Polymorphismus, „copy-number polymorphism“ (CNP) genannt, darstellen.

Grundsätzlich gilt, dass die Wahrscheinlichkeit für ein illegales meiotisches Rekombinationsereignis umso höher ist, wenn die duplizierten Segmente zwischen 10-400 kb groß sind und eine Homologie von über $96 \%$ Sequenzidentität haben. Eine mit 1:3000 Geburten häufige Mikrodeletion sind das velokardiofaziale (VCF) und DiGeorge- (CATCH22) Syndrom, deren flankierende duplizierte Segmente über $300 \mathrm{~kb}$ groß sind, mit einer Sequenzidentität von $99,7 \%$.

\section{Literatur}

Stankiewicz P et al (2012) Recurrent deletions and reciprocal duplications of $10 \mathrm{q} 11.21 \mathrm{q} 11.23$ including CHAT and SLC18A3 are likely mediated by complex low-copy repeats. Hum Mutat 33:165-179 